\title{
The Efficacy and Safety of Rivaroxaban in Preventing Deep Vein Thrombosis after Hip Replacement
}

L.WING. G.DANG AND Y. MIN*

Department of Orthopaedics, The Yijishan Hospital of Wannan Medical College, Anhui, People's Republic of China

Wang et al.: Efficacy and Safety Analysis of Rivaroxaban 
To evaluate the efficacy and safety of rivaroxaban versus low molecular weight heparin for the prevention of deep vein thrombosis after hip arthroplasty, 30 patients with primary unilateral hip arthroplasty in the Yijishan Hospital of Wannan Medical College between January 2017 and December 2018 were selected and randomly divided into the rivaroxaban group and the low molecular weight heparin group. Treatments started $6 \mathrm{~h}$ after replacement, the patients in the rivaroxaban group received rivaroxaban $10 \mathrm{mg} / \mathrm{d} \mathrm{for} 5$ $\mathrm{w}$ and the another group received low molecular weight heparin $4100 \mathrm{U} / \mathrm{d}$ for $2 \mathrm{w}$. Results indicated that full course of rivaroxaban demonstrated distinct efficacy with safety in preventing deep vein thrombosis after hip arthroplasty.

Key words: Deep vein thrombosis (DVT), low molecular weight heparin (LMWH), rivaroxaban

Venous thromboembolism is a common disease and deep vein thrombosis (DVT) in lower limbs is a vascular surgery disease, which often occurs in people post-surgery or in bedridden people with chronic disease, as well as people with limb disorders. DVT in lower limbs may puff up the infected limbs, leading to impaired mobility and even disability, and pulmonary artery embolism secondary to emboli detachment is more life-threatening ${ }^{[1]}$.

Because of the postsurgical trauma and reduced activity of patients due to long confinement to bed, the incidence of DVT after the hip operation is very high, up to 40$60 \%$, and pulmonary artery embolism secondary to hip operation becomes the major cause of death after orthopaedic operation ${ }^{[2]}$. With the development of medical technology and the availability of better prosthetic joints, artificial hip replacement has already become an important means to reshape hips in adult patients ${ }^{[3]}$. Therefore prevention of DVT in patients after hip replacement is increasingly important and became the focus of medical research both in China and abroad.

The China Guidance Consensus for prevention of venous thromboembolism after orthopaedic surgery suggests the following measures to prevent DVT, being gentle and careful during joint replacement to avoid venous intimal injury, using tourniquet according to criterion, uplifting infected limbs after joint replacement to avoid reflux disturbance, encouraging patients to make frequent turn-over, making moderate fluid infusion after joint replacement and so on. Use of venous pump of pelma, interval air inflation and pressure device, graded pressure stretch socks, Preventing DVT with drug treatment, which is the most important measure. A large number of clinical reports in China and abroad indicated the necessity of using drugs to prevent DVT after hip joint replacement ${ }^{[4-6]}$.

*Address for correspondence

E-mail: yjsyygk@sohu.com

Special Issue 3, 2020

Indian Journal of Pharmaceutical Sciences

12
The most commonly used drug to prevent DVT in China is low molecular weight heparin (LMWH), and there is enough clinical evidence reported both in China and abroad that LMWH can effectively reduce the incidence of DVT with high safety after hip joint replacement. However, the incidence of DVT after hip joint replacement is still high due to the limitation of the mode of administration, which causes poor compliance of patients who have to rely on hospitals for getting a full course of treatment once they are out of hospital.

The new generation orally active factor Xa inhibitor, rivaroxaban was reported to exert high efficacy and safety in preventing DVT in lower limbs when taken for full course of treatment. American College of Chest Physicians pointed out in 2012 that extending anticoagulation treatment course to $5 \mathrm{w}$ has better rate of preventing DVT and pulmonary artery embolism, reducing unexpected morbidity post-surgery. In China some clinical reports indicated the effect of 35 $\mathrm{d}$ treatment course and on account of the limitation of LMWH, the advantages of taking orally active rivaroxaban are highly evident. Combined with the actual medical situation in China, experiments were carried out on patients who received single-sided hip joint replacement in the Yijishan Hospital of Wannan Medical College from January 2017 and December 2018 to compare the effects of LMWH and rivaroxaban in preventing DVT after hip joint replacement as well as the safety of taking rivaroxaban for full treatment course.

Surgery of the patients of rivaroxaban and LMWH group was performed by the same group of surgeons, hip replacement approach was from outside of the hip side. All patients underwent ankle exercise including flexion and extension $1 \mathrm{~d}$ after hip replacement. All patients underwent hip and knee exercise $3 \mathrm{~d}$ after hip replacement. 
Patients in the rivaroxaban group received $6 \mathrm{~h}$ after hip replacement $10 \mathrm{mg} / \mathrm{d}$ rivaroxaban for $5 \mathrm{w}$. Patients in the LMWH group received $6 \mathrm{~h}$ after hip replacement, $4100 \mathrm{U} / \mathrm{d}$, for $2 \mathrm{w}$.

All patients were followed-up every $1 \mathrm{w}$ for 1 mo after hip replacement. Patients would return if they show any DVT symptoms to get a colour Doppler ultrasound check. Those who are symptomless would also get colour Doppler ultrasound examination at 2 and $5 \mathrm{w}$ after hip replacement. Assessment for DVT of lower limbs was carried out after hip replacement and further follow-up 6 mo after replacement.

The criteria were venous lumen with low intraluminal echo or no echo; thrombotic static segment; no blood flow within the vein completely or only a small amount of blood flow and Pulse Chong Doppler showed no flow or spectrum does not change with respiration.

The safety assessment included haemoglobin, platelets and coagulation tests before and after initiating drug treatment after hip replacement. Venous blood samples $3 \mathrm{~d}$ before hip replacement were collected from patients of the 2 groups. Venous blood samples at 2 and $5 \mathrm{w}$ after hip replacement were collected from patients of the rivaroxaban and LMWH group. Haemoglobin content, platelet count, prothrombin time, activated partial thromboplastin time values before and after the hip replacement were evaluated and compared. Drainage fluid of less than $10 \mathrm{ml} / \mathrm{d}$ was before extubation after hip replacement with all patients in the rivaroxaban and LMWH groups. Postoperative ecchymosis area was measured using the method of calculating total burn surface area as a \% of body surface area and the maximum ecchymosis area was recorded. The efficacy of rivaroxaban and LMWH in providing DVT prophylaxis after hip replacement as well as the safety of the treatment was compared. The tests were carried out based on the ethics approval with the Wannan Medical College of the ethics committee, and have obtained consent to publish from the participant.

SPSS 13.0 software (SPSS American public Division) for data analysis, experimental data are expressed as mean \pm standard deviation, T-test and the chi-square test were used to determine statistical significance at the probability level of $\mathrm{p}<0.05$.

Quantitative analytical comparison of experimental subjects and clinical data were made. Processing and analysis of all 50 cases of hip replacement patients were the result of Analysis, without any loss. Patients in the rivaroxaban and the LMWH group who underwent hip replacement had no significant differences among them in terms of sex, age and body mass index $(p>0.05)$ as shown in Table 1.

Rivaroxaban treatment prevented development of DVT in patients who underwent hip replacement therapy from. Treatment with rivaroxaban in hip replacement patients after discharge, review and regular follow-up after discharge showed that DVT did not occur not any symptoms of DVT could be observed; In the LMWHtreated patients who underwent total hip replacement, DVT occurred in 7 cases (28\%) during $2 \mathrm{w}$ after hip replacement to 6 mo follow-up period; of these 5 cases were of popliteal vein embolism, 1 case of femoral vein thrombosis and 1 case of iliac vein thrombosis. The results indicated that rivaroxaban treatment in patients with hip replacement caused significantly lower incidence of DVT compared to those in the LMWH group $(\mathrm{p}<0.05)$, Table 2 .

The safety of rivaroxaban treatment in preventing DVT hip replacement patients is shown in Table 3 and 4 . Table 3 showed that there was no severe bleeding or large wound hematoma occurred in rivaroxaban and LMWH group after hip arthroplasty and there was no significant difference $(p>0.05)$ between these two groups in terms of postoperative drainage or area of ecchymosis.

Table 4 showed that rivaroxaban and LMWH treated patients with hip replacement surgery $3 \mathrm{~d}$ before joint replacement and $5 \mathrm{~W}$ after hip replacement

TABLE 1: COMPARISON OF PATIENTS UNDERWENT HIP ARTHROPLASTY IN THE RIVAROXABAN GROUP AND THE LMWH GROUP

\begin{tabular}{lccc}
\hline Project & $\begin{array}{c}\text { Rivaroxaban } \\
\text { group }\end{array}$ & LMWH group & $\mathrm{p}$ \\
\hline $\begin{array}{l}\text { Sex ratio } \\
\text { (male:female) }\end{array}$ & $13: 12$ & $13: 12$ & 0.585 \\
$\begin{array}{l}\text { Age }(\mathrm{y}) \\
\begin{array}{l}\text { Body mass index } \\
\left(\mathrm{kg} / \mathrm{m}^{2}\right)\end{array}\end{array}$ & $65.40 \pm 5.26$ & $63.70 \pm 5.57$ & 0.609 \\
\hline
\end{tabular}

Clinical information of patients underwent hip arthroplasty in the rivaroxaban group and the low molecular weight heparin (LMWH) group (each group has $\mathrm{N}=25$ ). The differences of gender, age, body mass index between both groups was not significant $(p>0.05)$

TABLE 2: INCIDENCE OF DVT IN PATIENTS TREATED WITH RIVAROXABAN OR LMWH AFTER HIP ARTHROPLASTY

\begin{tabular}{lccc}
\hline Project & $\begin{array}{c}\text { Rivaroxaban } \\
\text { group }\end{array}$ & $\begin{array}{c}\text { LMWH } \\
\text { group }\end{array}$ & p \\
\hline The number of cases of DVT & 0 & 7 & - \\
The incidence of DVT (\%) & 0 & 28 & 0.019 \\
\hline
\end{tabular}

The incidence of deep vein thrombosis (DVT) in the rivaroxaban group was significantly lower than that in low molecular weight heparin (LMWH) group. Each group has 25 patients 
for the rivaroxaban-treated group and $2 \mathrm{w}$ after hip replacement for the LMWH-treated group had similar levels of haemoglobin and platelets, and prothrombin and activated partial thromboplastin times, with no significant differences among groups $(\mathrm{p}>0.05)$. Hip replacement instructions indicated that applications of prompt treatment for prevention of DVT has a clear effect and application of sufficient course of treatment with rivaroxaban is associated with safety.

Venous thromboembolism syndrome is a clinically common disease of vascular surgery, including DVT and pulmonary artery thromboembolism, the main reasons being high blood viscosity, slow blood flow and injury to vascular wall and it often occurs after surgeries of various kinds and to bedridden patients with chronic diseases as well as people with limb disorders ${ }^{[7-10]}$.

Hip orthopaedic surgery slows down venous blood flow due to reasons such as the long-time restraining of limbs, twisting of joints during the surgery, oppressing from tourniquet and long bedridden hours after the surgery; surgical trauma and traction lead to intimal injury of veins. These reasons contribute to cases of DVT after surgeries, while pulmonary artery embolism, which is often secondary to proximal DVT, becomes the main cause of unexpected deaths after orthopaedic surgeries ${ }^{[9,11]}$.

According to literature, incidences of DVT were as high as $42-57 \%$ after replacement of hip joint, incidence of

\section{TABLE 3: DRAINAGE FLUID VOLUME AND SUBCUTANEOUS ECCHYMOSIS AREA OF PATIENTS IN THE RIVAROXABAN AND THE LMWH GROUP}

\begin{tabular}{lcc}
\hline Group & $\begin{array}{c}\text { Postoperative } \\
\text { drainage }(\mathrm{ml})\end{array}$ & $\begin{array}{c}\text { Ecchymosis area } \\
\mathbf{( \% )}\end{array}$ \\
\hline Rivaroxaban group & $201.96 \pm 28.99$ & $0.76 \pm 0.59$ \\
LMWH group & $209.10 \pm 32.98$ & $0.80 \pm 0.61$ \\
$P$ & 0.221 & 0.523 \\
\hline
\end{tabular}

Drainage fluid volume or subcutaneous ecchymosis area were not different in patients with hip arthroplasty treated with either rivaroxaban or low molecular weight heparin (LMWH). Number of patients are 25 in each group and the data are expressed as mean \pm standard deviation. fatal pulmonary embolism being $0.1-0.2 \%$ and for the former, reports from China place the incidence as 47.1 $\%{ }^{[6,7]}$. Clinical researchers all over the world are trying hard to seek an effective way to reduce the incidence of DVT thereby to reduce morbidity and mortality due to secondary pulmonary artery embolism. During the extensive work to prevent DVT, drug prophylaxis was found to be great importance in preventing DVT after orthopaedic surgeries.

Heparin is an anticoagulant and a polymer made up of two alternated polysaccharoses and it has anticoagulatory effect both in vivo and in vitro. Clinically it is mainly used for thromboembolic disease, myocardial infarction, cardiovascular surgery, inspection of cardiac catheter, extracorporeal circulation, haemodialysis and many more. With the development of pharmacology and clinical medicine, application of heparin has increased continuously.

LMWH can bind to antithrombin III, and inactivate activated blood coagulation factors such as IIa, Xa, IXa and XIIa. It needs the involvement of antithrombin III to exert its anticoagulation effect, selectively killing the activity of coagulation factors; on the other hand, most part of heparin molecules can be integrated with blood platelet, inhibiting the aggregation of blood platelet, and low molecular heparin cannot be easily neutralized by the IV blood platelet factor for it is a fragment of heparin with an average small relative molecular mass of 4000-5000 depolymerized by chemicals or enzymes, and its anticoagulation effect and fibrinolysis are able to be intensified while its effect in antiplatelet and inducing haemorrhage is greatly weakened ${ }^{[8,12,13]}$.

Studies reported abroad have shown that LMWH can reduce $70 \%$ of incidence of DVT after hip replacement and there was no obvious increase of severe haemorrhage ${ }^{[14,15]}$. The effect of LMWH was also very remarkable in clinical research reported in China. It greatly reduced the incidence of DVT and has been approved by clinicians for extensive clinical application. But at the same time, the objective of LMWH to prevent DVT cannot be guaranteed because

TABLE 4: HEMOGLOBIN AND PLATELET LEVELS, PROTHROMBIN AND ACTIVATED PARTIAL THROMBOPLASTIN TIME OF PATIENTS IN THE RIVAROXABAN AND THE LMWH GROUPS.

\begin{tabular}{lcccc}
\hline Group & $\begin{array}{c}\text { Hemoglobin } \\
\text { level }(\mathrm{g} / \mathrm{l})\end{array}$ & $\begin{array}{c}\text { Platelet } \\
\text { levels }\left(\times 10^{9} / \mathrm{l}\right)\end{array}$ & Prothrombin time $(\mathbf{s})$ & $\begin{array}{c}\text { Activated partial } \\
\text { thromboplastin time }(\mathbf{s})\end{array}$ \\
\hline Rivaroxaban group & $4.78 \pm 2.18$ & $15.89 \pm 8.98$ & $0.29 \pm 0.78$ & $0.55 \pm 1.15$ \\
LMWH group & $4.81 \pm 2.09$ & $15.79 \pm 6.99$ & $0.30 \pm 0.74$ & $0.57 \pm 0.92$ \\
$\mathrm{P}$ & 0.195 & 0.116 & 0.451 & 0.843 \\
\hline
\end{tabular}

The haemoglobin and platelet levels, prothrombin and activated partial thromboplastin time between the rivaroxaban group and the low molecular weight heparin (LMWH) group were not significantly different $(p>0.05)$ 
of the route of administration which is parenteral toute, which causes poor patient compliance due to the fact that the patients have to rely on hospitals even more and more often do not receive full course of treatment once they are out of hospital[12].

Rivaroxaban is an inhibitor of both free Factor Xa and Factor Xa bound in the prothrombinase complex, which is highly selective and competitive inhibitor of factor Xa and was approved for clinical use in China in 2009. It dose-dependently prolongs activated thromboplastin time and prothrombin time ${ }^{[16]}$. Rivaroxaban does not inhibit thrombin (activated factor II), and yet there is no evidence to show that it has influence on blood platelet, thus risk of haemorrhage is low and its safety is high.

Rivaroxaban is the first oral anticoagulation agent in the world with fixed dosage and with no requirement for surveillance and it is clinically convenient as it has fewer drug interactions with food and drugs and does not need coagulation test ${ }^{[17]}$. The essential difference between rivaroxaban and heparin is that the former can directly antagonize the dissociation and combination of Xa factors without the involvement of antithrombin III while the latter needs antithrombin III for exerting its effects and it would be ineffective once the factor Xa and prothrombin complex has been formed. Combining experiments with China's actual clinical situations, a clinical test of $5 \mathrm{w}$ full course of treatment was carried out to rivaroxaban group; due to the limitations of LMWH, it was used for only $2 \mathrm{w}$ in hospital to compare these 2 methods of prevention of DVT in the clinic to identify the best treatment of DVT.

Results obtained showed that both rivaroxaban and LMWH were effective in preventing DVT after hip replacement and there was no incidence of DVT during the full course of treatment of $35 \mathrm{~d}$ with rivaroxaban. However, in the LMWH group, 7 cases of DVT occurred with an incidence of $28 \%$, and the difference in incidence of DVT in the two groups is significant $(p<0.05)$, the incidence of DVT for rivaroxaban group with $35 \mathrm{~d}$ of treatment is much lower than that of the LMWH group.

There is no obvious difference between the two groups before and after hip replacement in haemoglobin and platelet levels, prothrombin and activated partial thromboplastin time, as well as drainage volume and in the proportion of subcutaneous ecchymosis. The safety of rivaroxaban treatment equals that of LMWH

In general, as an oral drug to prevent DVT after orthopaedic surgeries, rivaroxaban is more effective when the full course of treatment was taken which obviously reduced the incidence of DVT after hip replacement and this fact makes it a more acceptable therapy to control DVT after hip replacement. Table 4 showed that there were no differences in the haemoglobin and platelet levels, prothrombin time an activated partial thromboplastin time $3 \mathrm{~d}$ before hip replacement between the rivaroxaban group and LMWH group. And there was no significance between rivaroxaban group at $5 \mathrm{w}$ after hip replacement and LMWH group at 2 $\mathrm{w}$ after hip replacement $(\mathrm{p}>0.05)$. The data show that rivaroxaban in the prevention of DVT have a clear effect, and sufficient course application rivaroxaban have reliable security.

\section{Financial interest and scholarship:}

Anhui Provincial Natural Science Foundation (1708085QH209).

\section{Conflict of interest}

The authors declare that they have no conflict of interest.

\section{REFERENCES}

1. Jiang P, Liu JL, Jia W. Incentive analysis of deep venous thrombosis leading to pulmonary embolism. Chin Med J 2012;92:3224-6.

2. Hunt JM, Bull TM. Clinical review of pulmonary embolism: Diagnosis, prognosis, and treatment. Med Clin 2011;95:120322.

3. Lachiewicz PF, Soileau ES. Changing indications for revision total hip arthroplasty. J Surg Orthop Adv 2005;14:82-4.

4. Qiu GX. The prevention guidelines venous thromboembolism in China major orthopedic surgery. Chin Joint Surg 2009;3:3803.

5. Jiang Y, Du H, Liu J, Zhou Y. Aspirin combined with mechanical measures to prevent venous thromboembolism after total knee arthroplasty: a randomized controlled trial. Chin Med J 2014;127:2201-5.

6. Qiu GX, Dai KR, Yang QM. The major orthopedic surgery expert advice in prevention of deep vein thrombosis. Chin Clinic Doct 2006;34:27-8.

7. Lv HS, Xu B. Deep vein thrombosis after artificial joint replacement surgery of. Chin Orthop J 1999;19:155-160.

8. Lieberman JR, Hsu WK. Prevention of venous thromboembolic disease after total hip and knee arthroplasty. JBJS 2005;87:2097-112.

9. Lin HQ, Gu Q, He XW. Analysis and discussion of colour Doppler ultrasound diagnosis of deep venous thrombosis. Chin Med Innovat 2012;7:157-8.

10. Ma L, Wang X, Jiang LH. Factors and prevention strategies for the deep vein thrombosis formation after total hip replacement. Chin J Clin Rehab Tissue Eng 2010;14:1677-80.

11. Parisi R, Visona A, Camporese G, Verlato F, Lessiani G, Antignani PL, et al. Isolated distal deep vein thrombosis: 
efficacy and safety of a protocol of treatment. Treatment of Isolated Calf Thrombosis (TICT) Study. Int Angiol 2009;28:68.

12. Weitz JI, Hirsh J, Samama MM. New antithrombotic drugs: American College of Chest Physicians evidence-based clinical practice guidelines. Chest 2008;133:234-56.

13. Nong L. Clinical analysis of rivaroxaban in prevention of deep vein thrombosis after total hip arthroplasty. Chin Health Indus 2011;12:63-4.

14. Kanaan AO, Silva MA, Donovan JL, Roy T, Al-Homsi AS. Meta-analysis of venous thromboembolism prophylaxis in medically ill patients. Clin Therap 2007;29:2395-405.

15. Geerts WH, Bergqvist D, Pineo GF, Heit JA, Samama CM, Lassen MR, et al. Prevention of venous thromboembolism: American College of Chest Physicians evidence-based clinical practice guidelines. Chest 2008;133:381-453.

16. Hull RD, Pineo GF, Stein PD, Mah AF, MacIsaac SM, Dahl OE, et al. Extended out-of-hospital low-molecular-weight heparin prophylaxis against deep venous thrombosis in patients after elective hip arthroplasty: A systematic review. Annals Int Med 2001;135:858-69.

17. Chen ZJ, Wang JW, Ren YJ. A Comparative Study of Prevention of rivaroxaban with low molecular weight heparin in primary total hip arthroplasty postoperative deep vein thrombosis. Chin J Orthop Trauma 2012;6:480-3.

This is an open access article distributed under the terms of the Creative Commons Attribution-NonCommercial-ShareAlike 3.0 License, which allows others to remix, tweak, and build upon the work non-commercially, as long as the author is credited and the new creations are licensed under the identical terms

This article was originally published in a special issue, "Biomedical research applications in Pharmaceutical Sciences"

Indian J Pharm Sci 2020:82(2)Spl issue3;11-16 